\title{
Coordinating a Supply Chain with Demand Disruption under o2o Business Model
}

\author{
Jun Zhang \\ Logistics Engineering and Management Faculty \\ Kunming Metallurgy College \\ Kunming, China \\ zjun434@sina.com.cn
}

\author{
Hong Chen \\ School of Management and Economics \\ University of Electronic Science and Technology of China \\ Chengdu, China \\ chenh@uestc.edu.cn
}

\begin{abstract}
A supply chain under o2o business model, a new kind of supply chain, is studied in this paper, in which the supply chain is comprised of one supplier (she) and multiple o2o retailers. The supplier observes that demand disruption takes place after her production plan is formulated. How to optimize the total supply chain profit in the centralized and the decentralized decisions is analyzed by using contract theory and game theory, which is also a new problem compared with the previous studies. The results obtained are as follows. In the centralized decision, the supplier needs to adjust the channel price if the disruption satisfies a given condition. If the disruption satisfies other conditions, the supplier needs to adjust both the channel retail price and the production quantity. In the decentralized decision, an improved revenue-sharing contract, a new supply contract, can coordinate the disrupted supply chain.
\end{abstract}

Keywords-o2o business model; supply chain; demand disruption; Stackelberg game

\section{INTRODUCTION}

The development of e-commerce grows rapidly around the world in recent years. E-commerce profoundly changes customers' consumption habit, which also has a great impact on the production and operation of traditional manufacturers and retailers. In this setting, o2o (Online to Offline/Offline to Online) business model, a new business model, is developed by some high-tech companies. They suggest that companies in the future should integrate online business with offline business. With the popularization of information technology, o2o business model becomes localized and integrated with mobile devices at the end of 2013, which means a new era of o2o business model is coming. For example, Tmall, an eretailer affiliated to Alibaba, begins to cooperate with Intime Department Store in 2013. Many e-retailers in China become o2o retailers when they sell products online and improve customers' shopping experience in their online and offline channels. E-commerce companies which include Taobao, Tmall, Amazon, Dangdang and Jingdong Shopping Center are o2o retailers. They are exactly the objects we focus on.

However, disruptions such as public health events, natural disasters and machine faults can influence supply chain operation. An initially-coordinated supply chain cannot be coordinated because of some disruptions, and whether supply chain members can survive or not is influenced by other disruptions. For example, the spread of plague caused by

The research is sponsored by National Natural Science Foundation of China (71472026) and Research Fund of Kunming Metallurgy College (14B005).
H7N9 in China makes the demand of chicken for food decrease dramatically. The spread of foot-and-mouth disease makes the demand for beef in Europe decrease dramatically, which influences the operation of beef supply chain in Europe. With the development of e-commerce, shopping online is widely spread throughout China. According to some statistics, the total sales volume in Tmall is about 120.7 billion RMB on 11th November 2016, which greatly influences the operation of its online and offline supply chain system. The e-retailers need to preorder a large number of products in order to meet the huge coming demand, and the logistics system is greatly affected by activities such as storing, transporting and distributing.

As it can be seen above, supply chain profit, supply chain operation and supply chain members' decisions can be affected by different disruptions. The influence is so serious that it is important to discuss how to coordinate supply chain when disruptions happen. This problem has been paid much attention to by scholars and companies worldwide.

\section{LITERATURE REVIEW}

Current research related to o2o business model focuses on recommendation system and how to improve e-commerce service. Tsai et al. find that retailers implementing o2o business model can better understand their customers [1]. Chen et al. believe that improvements in recommendation system can attract more online customers [2]. Hong examines the role of o2o business model in the marketing of local fresh produce [3]. Zhao analyzes the o2o business model in Wanda Group, a Chinese leading real estate company [4].

Studies closely related to this paper are called as disruption management. Clausen et al. firstly put forward disruption management [5]. How to coordinate supply chain with different disruptions is discussed by Qi et al. [6], Xu et al. [7, 8] and Huang et al. [9]. Lei et al. examine how to coordinate a one-supplier-one-retailer supply chain under asymmetric information with a linear contract [10]. Zhang et al. investigate different two-echelon supply chains under o2o business model, respectively $[11,12]$.

Compared with previous studies, there are some differences in this paper. Firstly, a one-supplier-multiple-o2oretailers supply chain under o2o business model, a new kind of supply chain, is established. Secondly, although the impact of 
the demand disruption on supply chain coordination is analyzed in this paper, the problem discussed is different from Zhang et al. Thirdly, this paper examines the role of the revenue-sharing contract in how to coordinate a disrupted supply chain system, which is also different from Xu et al., Qi et al., Huang et al. and Zhang et al.

\section{BENCHMARK MODEL}

A supply chain system comprising of one supplier (she) and multiple o2o retailers is studied in this section. The supplier is the price leader, and she controls the wholesale price and the revenue allocation ratio $\phi$. Those o2o retailers are the price followers. The transaction between the supplier and those retailers is realized under symmetric information. The number of o2o retailers is $N$. The supplier sells a kind of short-life-cycle product to each retailer according to her market forecast. Each retailer sells the product to consumers by using o2o e-commerce platform and customers evaluate the online shopping experience at the platform after they receive the product offline. Each retailer outsources his product distribution to a third-party logistics company. Those o2o retailers decide whether or not to sell the product according to the revenue-sharing contract the supplier offers.

Suppose that $p_{i}$ is the channel retail price in each segmented market and the demand function that the $i$-th o2o retailer faces in each segmented market is a nonlinear function, i.e., $d_{i}=D_{i} p_{i}^{-2 k_{i}}(i=1,2, \ldots, N) \cdot D_{i}$ is the potential market scale in the $i$-th market and $c_{s}$ is the supplier's unit production cost. Each retailer outsources his distribution. Each retailer's unit sales cost is $c_{r i}$ which includes the unit cost of using o2o ecommerce platform and the unit cost of distributing his product. $p_{i}$ is the unit retail price in the $i$-th sales market and $k_{i}\left(k_{i}>0\right)$ is the price sensitivity coefficient in the $i$-th sales market. $Q_{i}$ is the realized demand at the retail price, $p_{i}$. Then, the realized demand is $Q_{i}=D_{i} p_{i}^{-2 k_{i}}$ and the retail price is $p_{i}=\left(\frac{D_{i}}{Q_{i}}\right)^{\frac{1}{2 k_{i}}}$. The total profit of the supply chain system is

$$
\bar{f}^{T}\left(Q_{i}\right)=\sum_{i=1}^{N} Q_{i}\left[\left(\frac{D_{i}}{Q_{i}}\right)^{\frac{1}{2 k_{i}}}-c_{s}-c_{r i}\right] .
$$

From the first-order optimality condition, we obtain that the optimal channel retail price in each segmented market is

$$
\bar{p}_{i}=\frac{2 k_{i}\left(c_{s}+c_{r i}\right)}{2 k_{i}-1}
$$

the optimal sales quantity in each segmented market is

$$
\overline{Q_{i}}=D_{i}\left[\frac{2 k_{i}-1}{2 k_{i}\left(c_{s}+c_{r i}\right)}\right]^{2 k_{i}}
$$

and the optimal total supply chain profit is

$$
\bar{f}_{\max }^{T}\left(\overline{Q_{i}}\right)=\sum_{i=1}^{N} \frac{D_{i}\left(c_{s}+c_{r i}\right)}{2 k_{i}-1}\left[\frac{2 k_{i}-1}{2 k_{i}\left(c_{s}+c_{r i}\right)}\right]^{2 k_{i}} .
$$

Lemma 1. If $f_{s}\left(\overline{Q_{i}}\right)=(1-\phi) \cdot \bar{f}_{\max }^{T}\left(\overline{Q_{i}}\right)$ with $0<\phi<1$ or $f_{r}\left(\overline{Q_{i}}\right)=\phi \cdot \bar{f}_{\max }^{T}\left(\overline{Q_{i}}\right)$ with $0<\phi<1$, the supply chain system comprising of one supplier and $N$ o2o retailers can be coordinated under the revenue-sharing contract, where the wholesale price is $W_{i}=\phi\left(c_{s}-\frac{1-\phi}{\phi} c_{r i}\right)(0<\phi<1) \cdot f_{s}\left(\overline{Q_{i}}\right)$ is the optimal total profit of the supplier and $f_{r}\left(\overline{Q_{i}}\right)$ is the optimal total profit of the $N$ o2o retailers.

PROOF. When $W_{i}=\phi\left(c_{s}-\frac{1-\phi}{\phi} c_{r i}\right)(0<\phi<1)$, the retailers' profit function under the revenue-sharing contract $\left(W_{i}, \phi\right)$ is shown below, where $f_{r i}\left(\overline{Q_{i}}\right)$ is the $i$-th o2o retailer's optimal profit.

$$
f_{r}\left(\overline{Q_{i}}\right)=\sum_{i=1}^{N} f_{r i}\left(\overline{Q_{i}}\right)=\sum_{i=1}^{N} \phi \bar{p}_{i} \overline{Q_{i}}-\sum_{i=1}^{N} W_{i} \overline{Q_{i}}-\sum_{i=1}^{N} c_{r i} \overline{Q_{i}}=\sum_{i=1}^{N} \phi\left(\bar{p}_{i}-c_{s}-c_{r i}\right) \overline{Q_{i}}=\phi \bar{f}_{\max }^{T}\left(\overline{Q_{i}}\right)
$$

The other part of the lemma can be proofed by using the similar method. Thus, the revenue-sharing contract $\left(W_{i}, \phi\right)$ can coordinate the supply chain system.

\section{CENTRALIZED DECISION MAKING IN THE O2O SUPPLY CHAIN WITH DEMAND DISRUPTIONS}

The supplier observes that the disruption of the potential market scale in each segmented market happens in the supply chain system after her production plan is formulated. The disruption is captured by the term of $\Delta D_{i}$, if and only if $D_{i}+\Delta D_{i}>0$, where $\Delta D_{i}$ is the disruption of the market scale. This ensures that the disruption is practical in the real world. The discussion followed is based on the above condition.

After the disruption takes place, the demand function in each segmented market is $d_{i}=\left(D_{i}+\Delta D_{i}\right) p_{i}^{-2 k_{i}}$. The corresponding market scale is $Q_{i}=\left(D_{i}+\Delta D_{i}\right) p_{i}^{-2 k_{i}}$ and the retail price is $p_{i}=\left(\frac{D_{i}+\Delta D_{i}}{Q_{i}}\right)^{\frac{1}{2 k_{i}}} \cdot b_{1}\left(b_{1}>0\right)$ is the extra increased unit cost due to increasing production quantity and $b_{2}\left(b_{2}>0\right)$ is the extra unit disposal cost due to selling the remained products in the secondary market at the price lower than the marginal production cost. To simplify the discussion followed, we put forward the following assumption. The impact of the disruption on each segmented market every o2o retailer faces is consistent, which means that each segmented market reacts consistently to the disruption. That is to say, if any given segmented market scale increases, then every segmented market scale and the total market scale increase. If any given segmented market size decreases, then every segmented market size and the overall market size decrease. Thus, the corresponding total supply chain profit function is written as

$$
f^{T}\left(Q_{i}\right)=\sum_{i=1}^{N} Q_{i}\left[\left(\frac{D_{i}+\Delta D_{i}}{Q_{i}}\right)^{\frac{1}{2 k_{i}}}-c_{s}-c_{r i}\right]-b_{1}\left(\sum_{i=1}^{N} Q_{i}-\sum_{i=1}^{N} \overline{Q_{i}}\right)^{+}-b_{2}\left(\sum_{i=1}^{N} \overline{Q_{i}}-\sum_{i=1}^{N} Q_{i}\right)^{+} \cdot
$$


To further investigate the impact of the disruption mentioned above on the initial production plan, we put forward Lemma 2 below.

Lemma 2. Suppose that $Q_{i}^{*}$ is the optimal sales quantity which maximizes the total supply chain profit function shown in Equation (5) after the demand disruption happens. The following results hold: if $\Delta D_{i}>0$, then $Q_{i}^{*} \geq \overline{Q_{i}}$; if $\Delta D_{i}<0$, then $Q_{i}^{*} \leq \overline{Q_{i}}$.

Lemma 2 shows the following results. When the demand disruption in each segmented market is larger than zero, the supplier needs to meet the enlarged market scale by increasing production quantity; while the demand disruption in each segmented market is less than zero, the supplier needs to meet the shrunk market scale by decreasing production quantity.

According to Lemma 2, if $\Delta D_{i}>0$, then $Q_{i}^{*} \geq \overline{Q_{i}}$. Thus, optimizing the total supply chain profit function $f^{T}\left(Q_{i}\right)$ is equal to optimize the strictly concave function

$$
f_{1}^{T}\left(Q_{i}\right)=\sum_{i=1}^{N} Q_{i}\left[\left(\frac{D_{i}+\Delta D_{i}}{Q_{i}}\right)^{\frac{1}{2 k_{i}}}-c_{s}-c_{r i}\right]-b_{1}\left(\sum_{i=1}^{N} Q_{i}-\sum_{i=1}^{N} \overline{Q_{i}}\right)
$$

subject to $Q_{i} \geq \overline{Q_{i}}$.

If $\Delta D_{i}<0$, then $Q_{i}^{*} \leq \overline{Q_{i}}$. Thus, optimizing the total supply chain profit function $f^{T}\left(Q_{i}\right)$ is equal to optimize the strictly concave function

$$
f_{2}^{T}\left(Q_{i}\right)=\sum_{i=1}^{N} Q_{i}\left[\left(\frac{D_{i}+\Delta D_{i}}{Q_{i}}\right)^{\frac{1}{2 k_{i}}}-c_{s}-c_{r i}\right]-b_{2}\left(\sum_{i=1}^{N} \overline{Q_{i}}-\sum_{i=1}^{N} Q_{i}\right)
$$

subject to $Q_{i} \leq \overline{Q_{i}}$.

By using the methodology similar to Xu et al., Qi et al. and Huang et al., Theorem 1 is obtained which shows the optimal decisions in the centralized o2o supply chain system when the demand disruption takes place.

Theorem 1. When the market scale in each segmented market changes and the demand function in each segmented market is $d_{i}=\left(D_{i}+\Delta D_{i}\right) p_{i}^{-2 k_{i}}$, the supplier, the decision-maker in the centralized o2o supply chain system, needs to adjust the corresponding channel retail price and the corresponding production quantity in order to maximize the total supply chain profit. According to different disruptions, the optimal channel retail price $p_{i}{ }^{*}$ in each segmented market and the optimal sales quantity $Q_{i}^{*}$ in each segmented market are shown as follows:

$$
p_{i}^{*}= \begin{cases}\bar{p}_{i}+\frac{2 k_{i} b_{1}}{2 k_{i}-1}, & \text { if } \Delta D_{i} \in R 1 \\ \bar{p}_{i}\left(1+\frac{\Delta D_{i}}{D_{i}}\right)^{\frac{1}{2 k_{i}}}, & \text { if } \Delta D_{i} \in R 2 \\ \bar{p}_{i}-\frac{2 k_{i} b_{2}}{2 k_{i}-1}, & \text { if } \Delta D_{i} \in R 3 .\end{cases}
$$

$$
Q_{i}^{*}=\left\{\begin{array}{l}
\left(D_{i}+\Delta D_{i}\right)\left[\frac{2 k_{i}-1}{2 k_{i}\left(c_{s}+c_{r i}+b_{1}\right)}\right]^{2 k_{i}}, \text { if } \Delta D_{i} \in R 1 ; \\
D_{i}\left[\frac{2 k_{i}-1}{2 k_{i}\left(c_{s}+c_{r i}\right)}\right]^{2 k_{i}}, \text { if } \Delta D_{i} \in R 2 ; \\
\left(D_{i}+\Delta D_{i}\right)\left[\frac{2 k_{i}-1}{2 k_{i}\left(c_{s}+c_{r i}-b_{2}\right)}\right]^{2 k_{i}}, \text { if } \Delta D_{i} \in R 3 .
\end{array}\right.
$$

$R 1, R 2$ and $R 3$ are shown below.

$$
\begin{aligned}
& R 1: \Delta D_{i}>D_{i}\left[\left(1+\frac{b_{1}}{c_{s}+c_{r i}}\right)^{2 k_{i}}-1\right] ; \\
& R 2: D_{i}\left[\left(1-\frac{b_{2}}{c_{s}+c_{r i}}\right)^{2 k_{i}}-1\right] \leq \Delta D_{i} \leq D_{i}\left[\left(1+\frac{b_{1}}{c_{s}+c_{r i}}\right)^{2 k_{i}}-1\right] ; \\
& R 3: \Delta D_{i}<D_{i}\left[\left(1-\frac{b_{2}}{c_{s}+c_{r i}}\right)^{2 k_{i}}-1\right] .
\end{aligned}
$$

Theorem 1 illustrates the following results. When the market scale in each segmented market change, there exists robustness in the supplier's original production plan. When $\Delta D_{i}$ is in a particular area, the supplier does not need to change the initial sales quantity in each segmented market, but she needs to change the channel retail price in each segmented market in order to compensate for the extra cost derived from the disruption. The channel retail price in each segmented market is related to the market scale disruption in each segmented market. If $\Delta D_{i}$ exceeds the particular area, the supplier needs to change both her initial sales quantity and the corresponding retail price in each segmented market according to the change of the corresponding market scale. It is also shown that the original revenue-sharing contract in Section 3 cannot coordinate the o2o supply chain when the disruption takes place, and we need to redesign a new supply contract in order to coordinate the supply chain system.

\section{COORDINATING THE DECENTRALIZED O2O SUPPLY CHAIN WITH THE DEMAND DISRUPTIONS}

In the centralized decision, when the market scale in each segmented market change, the optimal strategy for the $i$-th o2o retailer is to choose the retail price $p_{i}^{*}$ and the procurement quantity $Q_{i}^{*}$. In the decentralized decision, if the supply chain members sign an appropriate supply contract which also makes each o2o retailer choose $p_{i}^{*}$ and $Q_{i}^{*}$, then the supply capacity in the decentralized o2o supply chain system is equal to that in the centralized o2o supply chain system. This means that the supply chain is coordinated in the decentralized decision. The revenue-sharing contract is used to coordinate the decentralized o2o supply chain system.

Let $R\left(Q_{i}\right)=b_{1}\left(Q_{i}-\overline{Q_{i}}\right)^{+}+b_{2}\left(\overline{Q_{i}}-Q_{i}\right)^{+}$. Given a revenue allocation ratio $\phi(0<\phi<1)$, the supplier provides o2o retailer $i \quad(i=1, \cdots, N)$ with an improved revenue-sharing contract in which the wholesale price for the retailer to choose is $W_{i}\left(Q_{i}\right)=\phi\left[c_{s}-\frac{1-\phi}{\phi} c_{r i}+\frac{R\left(Q_{i}\right)}{Q_{i}}\right]$. 
Theorem 2. In the decentralized o2o supply chain, if the market scale in each segmented market change, the supply chain can be coordinated by the revenue-sharing contract $\left(W_{i}\left(Q_{i}\right), \phi\right)$, where the wholesale price for each o2o retailer is $W_{i}\left(Q_{i}\right)=\phi\left[c_{s}-\frac{1-\phi}{\phi} c_{r i}+\frac{R\left(Q_{i}\right)}{Q_{i}}\right]$. The optimal total supply chain profit can be allocated between the supplier and the $N$ 02o retailers in any ratio at the same time.

PROOF. Given a revenue allocation ratio $\phi(0<\phi<1)$, the retailers' total profit function under the contract is shown below.

$$
\begin{aligned}
f_{r}\left(Q_{i}^{*}\right) & =\sum_{i=1}^{N} f_{r i}\left(Q_{i}^{*}\right)=\sum_{i=1}^{N}\left[\phi p_{i}^{*} Q_{i}^{*}-W_{i}\left(Q_{i}^{*}\right) Q_{i}^{*}-c_{r i} Q_{i}^{*}\right] \\
& =\sum_{i=1}^{N}\left[\phi p_{i}^{*} Q_{i}^{*}-\phi\left(c_{s}+c_{r i}+\frac{R\left(Q_{i}^{*}\right)}{Q_{i}^{*}}\right) Q_{i}^{*}\right] \\
& =\phi \sum_{i=1}^{N}\left[p_{i}^{*} Q_{i}^{*}-\left(c_{s}+c_{r i}\right) Q_{i}^{*}-R\left(Q_{i}^{*}\right)\right]=\phi f^{T}\left(Q_{i}^{*}\right)
\end{aligned}
$$

Thus, the supply chain is coordinated and the optimal total channel profit can be allocated among the supply chain members in any allocation ratio by changing the parameter $\phi$. This means that the improved revenue-sharing contract $\left(W_{i}\left(Q_{i}\right), \phi\right)$ can coordinate the decentralized o2o supply chain system.

Furthermore, if $\Delta D_{i}=0$, then $b_{1}=b_{2}=0$. The total supply chain profit function in this scenario is $f^{T}\left(Q_{i}\right)=\bar{f}^{T}\left(Q_{i}\right)$ and the wholesale price is $W_{i}\left(Q_{i}\right)=W_{i}$, which means that the improved revenue-sharing contract $\left(W_{i}\left(Q_{i}\right), \phi\right)$ can coordinate the o2o supply chain system when the disruptions do not happen. In other words, there exists anti-disruption ability in the improved revenue-sharing contract.

\section{CONCLUSIONS}

This paper studies how to coordinate one-suppliermultiple-o2o-retailers supply chain both in the centralized decision and in the decentralized decision when the market scale change. In the centralized decision, the supplier needs to increase the production quantities in order to meet the increased market demand when the disruption of the market scale satisfies a given condition. The supplier needs to decrease the production quantities in order to meet the shrunk market demand when the disruption of the market scale satisfies other conditions. There exists robustness in the initial production quantity when making the centralized decision. In other words, when the market scale disruption satisfies a given condition, the supplier does not need to change the initial production quantity and she only needs to change the corresponding retail price in order to compensate for the disposal cost originated from the disruptions. Furthermore, she needs to change both the corresponding production quantity and the corresponding channel retail price if the market scale disruption is not in the particular interval. An improved revenue-sharing contract is used to coordinate the decentralized o2o supply chain, which means that the contract can make the total supply chain profit maximize.

The study in supply chain under o2o business model is a new branch and there are abundant research opportunities in the future. For example, it is interesting to coordinate a supply chain when the transaction information between the supply chain participants are asymmetric. Another direction is to study the problem which the demand function the retailer faces is different from what we examine in this paper.

\section{REFERENCES}

[1] Tse-Ming Tsai, Ping-Che Yang, et al., "Pilot study toward realizing social effect in o2o commerce services," Social Informatics, 2013, pp. 268-273.

[2] Yen-Chiu Chen, Hui-Ching Hsieh, et al., "Improved precision recommendation scheme by BPNN algorithm in o2o commerce,” IEEE 10th International Conference on e-Business Engineering, 2013, September 11-13, Coventry, United Kingdom.

[3] Wenchang Hong, "Quanzhou agricultural e-commerce platform based on o2o modes,” 2013 International Workshop on Computer Science in Sports, 2013, August 1-2, Wuhan, China.

[4] Shouxiang Zhao and Xuewei Zhang, "Research on the development of traditional retail trends based on o2o- Wanda Group as an example," 2014 International Conference of the Academy of International Business, 2014b, June 23-26, Vancouver, Canada.

[5] J. Clausen, J. Hansen, J. Larsen, et al., "Disruption management," OR/MS Today, 2001, pp. 40-43.

[6] Xiangtong Qi, Jonathan F. Bard, and Gang Yu, "Supply chain coordination with demand disruptions,” Omega, 2004, pp. 301-312.

[7] Minghui Xu, et al., "The demand disruption management problem for a supply chain system with nonlinear demand function,” Journal of Systems Science and Systems Engineering, 2003, pp. 82-97.

[8] Minghui Xu, et al., "Coordinating dyadic supply chains when production costs are disrupted,” IIE Transactions, 2006, pp. 765-775.

[9] Chongchao Huang, Gang Yu, Song Wang, et al. "Disruption management for supply chain coordination with exponential demand function,” Acta Mathematica Scientia, 2006, pp. 655-669.

[10] Dong Lei, Jianbin Li, and Zhixue Liu, "Supply chain contracts under demand and cost disruptions with asymmetric information," International Journal of Production Economics, 2012, pp. 116-126.

[11] Jun Zhang, Hong Chen, et al. "How to coordinate supply chain under o2o business model when demand deviation happens,” Management Science and Engineering, 2015, pp. 24-28.

[12] Jun Zhang, Hong Chen, Wuyi Zhang, "How to coordinate supply chain under o2o business model when demand deviation and retailer's sales cost deviation happen,” IEEE 13th International Conference on Service Systems and Service Management, 2016, June 24-26, Kunming, China.

[13] Abel P. Jeuland, and Steven M. Shugan, "Managing channel profits," Marketing Science, 1983, pp. 239-272.

[14] G.P. Cachon, M.A. Larivere, "Supply chain coordination with revenue sharing contracts: strengths and limitations,” Management Science, 2005, pp. 30-44.

[15] Paul R. Kleindorfer and Germaine H. Saad, "Managing disruption risks in supply chains,” Production and Operations Management, 2005, pp. 53-68. 